\title{
Optimization of ultrasound assessments of arterial function
}

\author{
Lee Stoner ${ }^{1,2 *}$, Cary West ${ }^{2}$, Danielle Morozewicz Cates ${ }^{2}$, Joanna M. Young ${ }^{3}$ \\ ${ }^{1}$ School of Sport and Exercise, Massey University, Wellington, New Zealand;
${ }^{2}$ Department of Kinesiology, University of Georgia, Ramsey Center, Athens, USA;
${ }^{3}$ Lipid and Diabetes Research Group, Diabetes Research Institute, Christchurch, New Zealand.
Email: ${ }^{\text {dr.1.stoner@gmail.com }}$
}

Received 16 September 2011; revised 18 November 2011; accepted 18 November 2011.

\section{ABSTRACT}

Ultrasound technology is widely used to make assessments of arterial function. The delicate nature of these measurements requires that sources of errors are limited. Therefore, the aim of this study was to assess variability due to probe selection and optimization settings. Methods: Ten healthy 20 - 26 year old male and female subjects were tested. Brachial artery size (diameter) was measured thirty times a second using a B-mode Ultrasound unit equipped with a high-resolution video capture device. Distension was calculated using systolic and diastolic diameters. To assess intersession variability, we made recordings over twelve minutes; with the probe being removed and re-positioned every four minutes. To assess variability due to probe selection and optimization, we manipulated four parameters: 1) Probe selection (7 $13 \mathrm{MHz}, 5$ - $10 \mathrm{MHz}, 6$ - 9 MHz); 2) Probe frequency (11 MHZ, 9.6 MHZ, 8 MHz); 3) Measurement location (near, center or middle field); And 4) Image mode (B-mode, duplex-mode). To assess inter-session variability, three sets of recordings were made for each probe selection and optimization setting. Results: Mean diameter ICC's for inter-session variability, probe frequency, measurement location, image display size, and probe selection were $0.99,0.98,0.97$, 0.99 , and 0.90 respectively. Distension ICC's for intersession variability, probe frequency, measurement location, image display size, and probe selection were $0.66,0.26,0.62,0.60$, and 0.51 respectively. Conclusions: Altering probe selection increases measurement variability to the greatest extent. However, as long as probe selection and optimization settings are kept constant, our inter-session variability shows that reliable measurements can be made.

Keywords: Ultrasound; Reproducibility; Diameters; Distension; Arterial Stiffness

\section{INTRODUCTION}

Ultrasound is widely used for the diagnostic assessment of the carotid and peripheral arteries. The elastic properties of carotid and peripheral arteries are assessed by studying dynamic properties of the arterial walls. Through measurement of arterial distention, together with local blood pressures, indices of arterial stiffness can be calculated. Assessments of arterial stiffness have shown to predict future cardiovascular complications [1-4].

In order to calculate arterial stiffness, the diameter of a given artery must be continuously measured across the cardiac cycle. For a carotid artery, this may entail measurements that range from $8.0 \mathrm{~mm}$ to $\sim 8.3 \mathrm{~mm}$, a distention of $0.3 \mathrm{~mm}$. For peripheral arteries, the distention range will be much lower. Therefore, even small variations in systolic or diastolic diameters can notably impact distention measurements. For this reason, it is important to limit possible sources of error. However, the requirement for standardization of ultrasound technical settings has not been reported in the literature.

The aim of this study was to assess variability due to probe selection and optimization settings. To assess variability due to probe selection and optimization four parameters were manipulated: 1) Probe selection; 2) Probe frequency; 3) Measurement location; And, 4) Image mode. To assess inter-session variability, three recordings were made for each parameter.

\section{METHODS}

\subsection{Subjects}

Ten healthy 20 - 26 year old male and female subjects were tested. Informed consent was obtained from the subjects after they were given a detailed description of the procedures. The study was approved by the University of Georgia Institutional Review Board. Subjects were excluded from the study if they demonstrated any cardiovascular disease health risks or were taking medications with known vasoactive properties. Subjects were 
asked to abstain from caffeine, high-fat foods, and alcohol for 24 hours prior to testing.

\subsection{Protocol}

Testing commenced following at least 20 minutes of quiet supine rest. All measurements for a given subject were made in one sitting. Brachial artery size (diameter) was measured using a B-mode Ultrasound unit equipped with a high-resolution video capture device. Diastolic, systolic and mean diameters were recorded. Recordings were made using eleven probe selection and optimization settings (see Table 1). To assess inter-session variability, three sets of recordings were made for each probe selection and optimization setting, with the probe being removed and re-positioned every four minutes. The three probes were linear array transducers. To compare probes the highest imaging frequencies were set for each probe (LA39, $11 \mathrm{MHz}$; 739, $9 \mathrm{MHz}$; 546, 6.6 MHz). Aside from the probe comparison measurements, the highest resolution probe (LA39) was used. Aside from the location measurements, images were focused on the center of the image display field. Aside from imaging mode measurements, B-mode was used. Care was taken to ensure that the same portion of the brachial artery was imaged for all measurements. Subjects were asked to hold their breath for ten seconds for each recording.

\subsection{Diameter Measurements}

High-resolution Brightness-mode (B-mode) ultrasound measurements were made using a GE 400CL duplex color Doppler unit (GE Medical, Milwaukee, Wisconsin). The brachial artery of the left arm was measured in the distal third of upper arm. Care was taken to ensure that the vessel clearly extended across the entire [un-zoomed] imaging plane to minimize the likelihood of skewing the vessel walls. Magnification and focal zone settings were then adjusted to optimize imaging of the proximal and distal vessel walls. The image was comprised of $400 \times$ 400 pixels over an area of $16 \times 16 \mathrm{~mm}$, with a pixel resolution of $0.04 \times 0.04 \mathrm{~mm}$. A specialized probe holding device enabled precise positioning and ensured that pressure on the artery was minimized. The precise position of the ultrasound probe was recorded and marked.

\subsection{Diameter Analysis}

Moving Picture Experts Group-2 (MPEG-2) recordings were captured using a Dell Laptop PC equipped with a video capture device (ADS technologies, Cerritos, California). Video files collected at 30 frames/second were converted to Joint Photographic Experts Group (JEPG) images and subsequently used to make 30 diameter measurements/second. JPEG images provide comparable accuracy for ultrasound image measurements compared
Table 1. Probe and optimization setting parameters.

\begin{tabular}{cc}
\hline Optimization Setting & Sub-Setting \\
\hline Linear Array Probe & $739(5-10 \mathrm{MHz})$ \\
& $549(6-9 \mathrm{MHz})$ \\
& $11 \mathrm{MHz}$ \\
Probe Frequency & $9.6 \mathrm{MHz}$ \\
& $8 \mathrm{MHz}$ \\
Field Location & Near Field \\
& Center field \\
Imaging Mode & Far Field \\
& B-Mode \\
& PD-Mode (duplex) \\
\hline
\end{tabular}

to the Digital Image and Communications in Medicine (DICOM) standard [5]. Images were measured offline using semi-automated edge-detection software custom written to interface with the LabVIEW data acquisition platform (version 8.1, National Instruments, Austin, Texas) [6,7]. Custom written Excel Visual Basic code was used to fit peaks and troughs to diameter waveforms in order to calculate diastolic, systolic, and mean diameters. The within-session SEM3,1 for diameter measurement with the described set-up is $0.046 \mathrm{~mm}$. The between-day coefficient of variation is $2.7 \%$ for resting diameter measurements [8].

\subsection{Statistical Analysis}

Statistical analysis was undertaken using SPSS 13 for windows (SPSS Inc, Chicago, IL). The single measures intra-class correlation coefficient (ICC) were calculated using a two-way mixed effects (absolute agreement) model where subject effects are random and the optimization/probe settings fixed. In general, values above 0.75 can be considered to represent excellent reliability, values between 0.4 and 0.75 represent fair to good reliability and values below 0.4 represent poor reliability [9].

Standard error of measurement (SEM) and respective confidence intervals were calculated using Eq. 1 and Eq. 2:

$$
\begin{gathered}
\mathrm{SEM}=\mathrm{SD} \times \sqrt{(1-\mathrm{ICC})} \\
95 \% \mathrm{CI}=\operatorname{Mean} \pm(\mathrm{SEM} \times 1.96)
\end{gathered}
$$

where $\mathrm{SD}=$ the sample standard deviation, and $\mathrm{ICC}=$ as calculated above.

Bland-Altman plots were constructed to provide an indication of systematic bias and random error [10,11]. 
The $95 \%$ confidence intervals of limits of agreement were calculated using Eq. 3:

$$
95 \%=d+1.96 \times \mathrm{SD}
$$

where: $d=$ the sample bias (mean difference), and $\mathrm{SD}=$ standard deviation of differences.

\section{RESULTS}

\subsection{Within-Session Variability}

Table 2 shows the inter-session variability for diameter measurements. Single measure ICC values for $D_{m}, D_{d}$, and $D_{s}$ show excellent reliability. The \%SEM is lowest for $D_{d}$ and highest for $D_{s}$. The Bland-Altman plot shown in Figure 3 compares trials 1 and 3. There was no indication of systemic bias across the three trials. The ICC for $\mathrm{D}_{\Delta}$ shows fair to good reliability. The \%SEM is notably higher for $\mathrm{D}_{\Delta}$ than for single diameters. BlandAltman plots for $\mathrm{D}_{\Delta}$ (not shown) do not indicate systemic bias over the three trials.

\subsection{Diameter Measurement Variability across Ultrasound Settings}

Table 3 shows the variability for diameter measurements across ultrasound settings. Single measure ICC values for $D_{m}, D_{d}$, and $D_{s}$ diameters show excellent reliability. The \%SEM is lowest for $D_{s}$ and highest for $D_{d}$. The ICC for $\mathrm{D}_{\Delta}$ shows poor reliability. The \%SEM is notably higher for $\mathrm{D}_{\Delta}$ than for systolic or diastolic diameters.

\subsection{Diameter Measurement Variability for Each Ultrasound Setting}

Table 4 shows the variability for diameter measurements for each ultrasound setting. Figures $\mathbf{1}$ and $\mathbf{2}$ show example diameter analysis and resultant waveforms for LA39 and 546 probes. ICC values for $\mathrm{D}_{\mathrm{m}}, \mathrm{D}_{\mathrm{d}}$, and $\mathrm{D}_{\mathrm{s}}$ across probe frequencies show excellent reliability. Bland-Altman plots (not shown) show a bias for smaller diameters as the probe frequency decreases.

The ICC for $\mathrm{D}_{\Delta}$ shows poor reliability. The $\% \mathrm{SEM}$ is notably higher for $\mathrm{D}_{\Delta}$ than for single diameters. The Bland-Altman plot shown in Figure 4 compares frequencies $11 \mathrm{MHz}$ and $8 \mathrm{MHz}$ for the LA39 probe. There was no indication of systemic bias across the three probe frequencies.

ICC values for $D_{m}, D_{d}$, and $D_{s}$ across measurement location show excellent reliability. Bland-Altman plots (not shown) show a bias for smaller diameters as the measurement location moves from the near- to the far-side of the ultrasound imaging field. The ICC for $\mathrm{D}_{\Delta}$ shows fair to good reliability. The \%SEM is notably higher for $\mathrm{D}_{\Delta}$ than for single diameters. Bland-Altman plots (not shown) show a bias for larger $\mathrm{D}_{\Delta}$ as the meas-
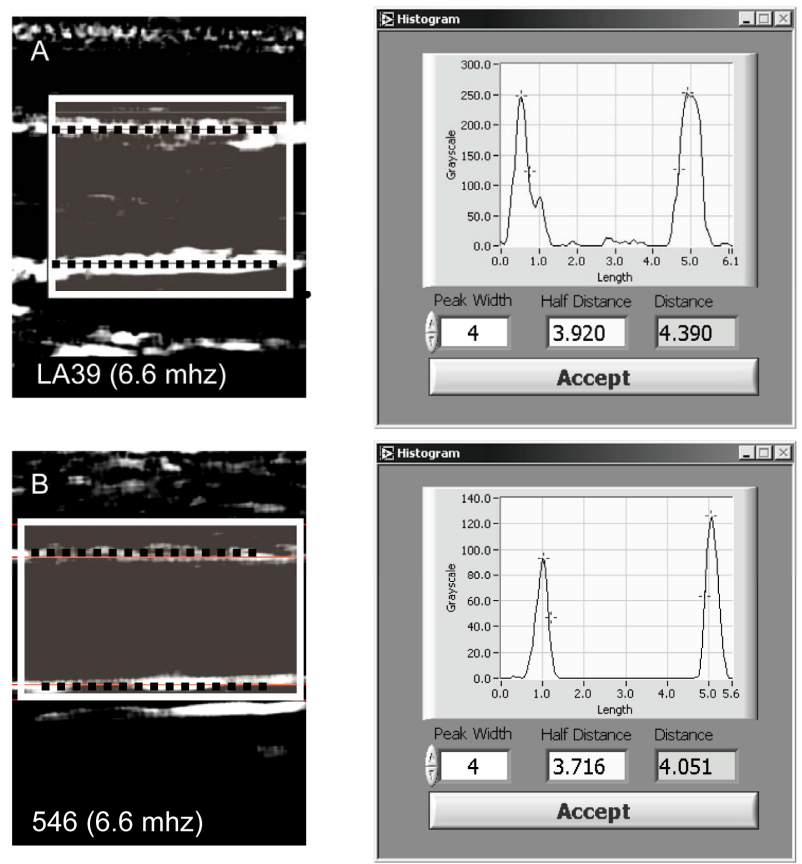

Figure 1. Examples of semi-automated diameter analysis on images collected from (A) LA39 (11 MHz) and (B) 546 (6.6 $\mathrm{MHz})$ probes. Images are captured at a rate of 30 images per second. The images are identical to those shown in Figure 2.

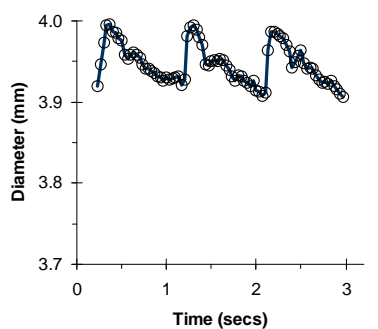

A. LA39 (11MHz)

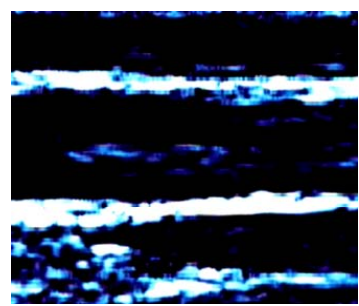

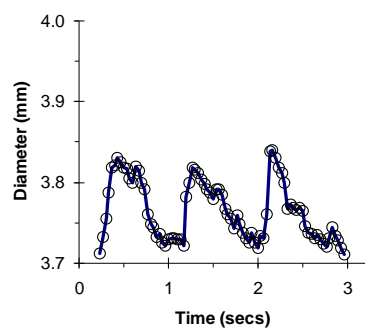

B. $546(6.6 \mathrm{MHz})$

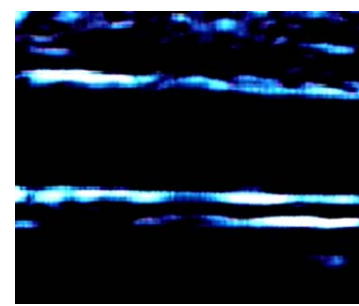

Figure 2. Example of images collected from (A) LA39 (11 $\mathrm{MHz})$ and (B) $546(6.6 \mathrm{MHz})$ probes. Images were taken during diastole and illustrate the first image used for analysis as shown by the corresponding graphs above the images.

urement location moves from the near- to the far-side of ultrasound imaging field.

ICC values for $D_{m}, D_{d}$, and $D_{s}$ across imaging mode show excellent reliability. Bland- Altman plots (not shown) show a bias for smaller diameters when imaging in $\mathrm{PD}$ versus $\mathrm{B}$-mode. The ICC for $\mathrm{D}_{\Delta}$ shows fair to 
good reliability. The \%SEM is notably higher for $\mathrm{D}_{\Delta}$ than for single diameters. Bland- Altman plots (not shown) show no indication of bias.

ICC values for $D_{m}, D_{d}$, and $D_{s}$ across probe selection show excellent reliability. Compared to the other optimization settings probe selection results in the lowest
ICC and highest \%SEM. The Bland-Altman plot shown in Figure 5 compares probes LA39 (11 MHz) and 539 (6.6 MHz). The 539 probe results in smaller diameters than for LA39 and $739(9 \mathrm{MHz})$ probes. The LA39 and 739 probes are more comparable. The ICC for $\mathrm{D}_{\Delta}$ shows fair to good reliability. The \%SEM is notably higher for

Table 2. Inter-session diameter measurement variability.

\begin{tabular}{ccccc}
\hline & $\mathrm{D}_{\mathrm{m}}$ & $\mathrm{D}_{\mathrm{s}}$ & $\mathrm{D}_{\mathrm{d}}$ & $\mathrm{D}_{\Delta}$ \\
\hline $\mathrm{T} 1(\mathrm{~mm})$ & $4.143(0.545)$ & $4.189(0.518)$ & $4.076(0.487)$ & $0.113(0.053)$ \\
$\mathrm{T} 2(\mathrm{~mm})$ & $4.149(0.493)$ & $4.196(0.495)$ & $4.076(0.492)$ & $0.120(0.054)$ \\
$\mathrm{T} 3(\mathrm{~mm})$ & $4.148(0.506)$ & $4.198(0.509)$ & $4.081(0.486)$ & $0.117(0.066)$ \\
Mean $(\mathrm{mm})$ & $4.147(0.513)$ & $4.194(0.506)$ & $4.078(0.488)$ & $0.117(0.050)$ \\
$\mathrm{ICC}$ & 0.992 & 0.991 & 0.996 & 0.661 \\
SEM $3,1(\mathrm{~mm})$ & 0.046 & 0.048 & 0.031 & 0.029 \\
$\mathrm{SEM}(\%)$ & 1.107 & 1.144 & 0.757 & 25.171 \\
LCI $(\mathrm{mm})$ & 4.057 & 4.100 & 4.017 & 0.059 \\
UCI $(\mathrm{mm})$ & 4.237 & 4.288 & 4.138 & 0.174 \\
\hline
\end{tabular}

Diastolic, systolic, and mean diameters, and distension for each four-minute interval. Values are mean (SD).

Table 3. Diameter measurement variability across ultrasound settings.

\begin{tabular}{|c|c|c|c|c|}
\hline & $\mathrm{D}_{\mathrm{m}}$ & $\mathrm{D}_{\mathrm{s}}$ & $\mathrm{D}_{\mathrm{d}}$ & $\mathrm{D}_{\Delta}$ \\
\hline LA39 (mm) & $4.121(0.504)$ & $4.161(0.505)$ & $4.089(0.504)$ & $0.072(0.043)$ \\
\hline $739(\mathrm{~mm})$ & $4.140(0.539)$ & $4.176(0.542)$ & $4.107(0.530)$ & $0.069(0.046)$ \\
\hline $546(\mathrm{~mm})$ & $4.040(0.605)$ & $4.101(0.604)$ & $4.002(0.619)$ & $0.099(0.044)$ \\
\hline $11 \mathrm{mhz}(\mathrm{mm})$ & $4.183(0.553)$ & $4.252(0.499)$ & $4.168(0.504)$ & $0.084(0.042)$ \\
\hline $9.6 \mathrm{mhz}(\mathrm{mm})$ & $4.127(0.516)$ & $4.191(0.514)$ & $4.090(0.510)$ & $0.101(0.064)$ \\
\hline $8.2 \mathrm{mhz}(\mathrm{mm})$ & $4.109(0.533)$ & $4.148(0.554)$ & $4.070(0.537)$ & $0.078(0.043)$ \\
\hline Left (mm) & $4.205(0.487)$ & $4.230(0.502)$ & $4.175(0.482)$ & $0.055(0.050)$ \\
\hline Cent. (mm) & $4.179(0.473)$ & $4.209(0.469)$ & $4.145(0.471)$ & $0.064(0.044)$ \\
\hline Right (mm) & $4.144(0.516)$ & $4.186(0.527)$ & $4.109(0.510)$ & $0.077(0.047)$ \\
\hline $\mathrm{B}(\mathrm{mm})$ & $4.182(0.476)$ & $4.226(0.477)$ & $4.156(0.481)$ & $0.071(0.042)$ \\
\hline $\mathrm{PD}(\mathrm{mm})$ & $4.151(0.501)$ & $4.191(0.521)$ & $4.127(0.501)$ & $0.064(0.067)$ \\
\hline Mean (mm) & $4.144(0.046)$ & $4.190(0.043)$ & $4.113(0.051)$ & $0.077(0.014)$ \\
\hline ICC & 0.915 & 0.918 & 0.914 & 0.372 \\
\hline $\mathrm{SEM}_{3,1}(\mathrm{~mm})$ & 0.013 & 0.012 & 0.015 & 0.011 \\
\hline SEM (\%) & 0.320 & 0.292 & 0.360 & 14.26 \\
\hline$L C I(\mathrm{~mm})$ & 4.118 & 4.166 & 4.084 & 0.055 \\
\hline$U C I(\mathrm{~mm})$ & 4.170 & 4.214 & 4.142 & 0.098 \\
\hline
\end{tabular}

Diastolic, systolic, and mean diameters, and distension for each ultrasound setting. Values are mean (SD). 
Table 4. Diameter measurement variability for each ultrasound setting.

\begin{tabular}{|c|c|c|c|c|c|c|}
\hline & & Mean (mm) & ICC & $\mathrm{SEM}_{3,1}(\mathrm{~mm})$ & SEM (\%) & $95 \% \mathrm{CI}(\mathrm{mm})$ \\
\hline \multirow{4}{*}{ Probe } & $\mathrm{D}_{\mathrm{m}}$ & $4.100(0.532)$ & 0.902 & 0.167 & 4.065 & $(3.774,4.427)$ \\
\hline & $\mathrm{D}_{\mathrm{s}}$ & $4.146(0.534)$ & 0.909 & 0.161 & 3.885 & $(3.830,4.462)$ \\
\hline & $D_{d}$ & $4.066(0.534)$ & 0.899 & 0.170 & 4.177 & $(3.733,4.399)$ \\
\hline & $\mathrm{D}_{\Delta}$ & $0.080(0.037)$ & 0.512 & 0.026 & 32.50 & $(0.029,0.131)$ \\
\hline \multirow{4}{*}{ Freq. } & $\mathrm{D}_{\mathrm{m}}$ & $4.140(0.531)$ & 0.980 & 0.075 & 1.815 & $(3.992,4.287)$ \\
\hline & $\mathrm{D}_{\mathrm{s}}$ & $4.197(0.519)$ & 0.970 & 0.090 & 2.141 & $(4.021,4.373)$ \\
\hline & $D_{d}$ & $4.109(0.514)$ & 0.975 & 0.081 & 1.980 & $(3.950,4.269)$ \\
\hline & $\mathrm{D}_{\Delta}$ & $0.088(0.036)$ & 0.255 & 0.031 & 35.26 & $(0.027,0.149)$ \\
\hline \multirow{4}{*}{ Location } & $\mathrm{D}_{\mathrm{m}}$ & $4.176(0.487)$ & 0.967 & 0.088 & 2.118 & $(4.003,4.349)$ \\
\hline & $\mathrm{D}_{\mathrm{s}}$ & $4.209(0.495)$ & 0.973 & 0.081 & 1.934 & $(4.049,4.368)$ \\
\hline & $\mathrm{D}_{\mathrm{d}}$ & $4.143(0.483)$ & 0.966 & 0.089 & 2.148 & $(3.969,4.318)$ \\
\hline & $\mathrm{D}_{\Delta}$ & $0.065(0.041)$ & 0.617 & 0.025 & 38.62 & $(0.016,0.115)$ \\
\hline \multirow{4}{*}{ Display } & $\mathrm{D}_{\mathrm{m}}$ & $4.166(0.488)$ & 0.994 & 0.038 & 0.908 & $(4.092,4.240)$ \\
\hline & $\mathrm{D}_{\mathrm{s}}$ & $4.219(0.497)$ & 0.986 & 0.059 & 1.393 & $(4.103,4.334)$ \\
\hline & $D_{d}$ & $4.145(0.491)$ & 0.995 & 0.035 & 0.838 & $(4.077,4.213)$ \\
\hline & $\mathrm{D}_{\Delta}$ & $0.073(0.051)$ & 0.596 & 0.032 & 43.83 & $(0.010,0.136)$ \\
\hline
\end{tabular}

Mean (SD) diameters for each ultrasound setting. Absolute difference (D), coefficients of variation (CV), intra-class correlation coefficient (ICC), and standard error of measurement (SEM) plus confidence intervals (CI) are shown.

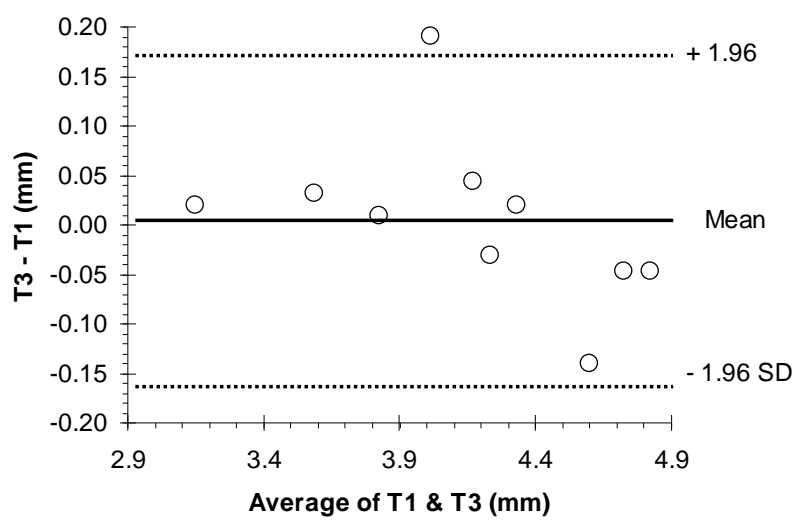

Figure 3. Bland-Altman plot of diameter difference values (Trial 3 - Trial 1) on average diameter values ((Trial $1+$ Trial $3) / 2)$ ). The mean difference, upper boundary ((mean + (SD of mean $\times 1.96))$, and lower boundary $(($ mean $-($ SD of mean $\times$ 1.96)) are shown.

$\mathrm{D}_{\Delta}$ than for single diameters. Bland-Altman plots (not shown) indicate bias towards larger $\mathrm{D}_{\Delta}$ for the 539 probe compared to the other two probes.

\section{DISCUSSION}

This study shows that arterial diameters can be reliably

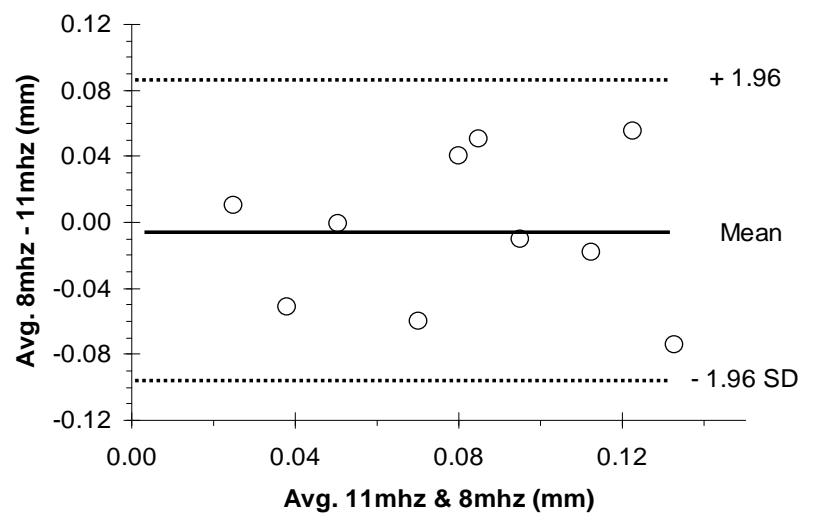

Figure 4. Bland-Altman plot of distension difference values (8 $\mathrm{MHz}-11 \mathrm{MHz})$ on average distension values $((11 \mathrm{MHz}+8$ $\mathrm{MHz}) / 2)$ ). The mean difference, upper boundary ((mean $+(\mathrm{SD}$ of mean $\times 1.9 .6))$, and lower boundary $(($ mean $-(\mathrm{SD}$ of mean $\times 1.96))$ are shown.

measured within-session, but that measurement error for arterial distention calculation is notably higher. Also, it was found that variations in ultrasound probe selection and optimization settings contribute to some measurement bias, though with little impact on the reliability of diameter measurements. However, variations in ultra- 


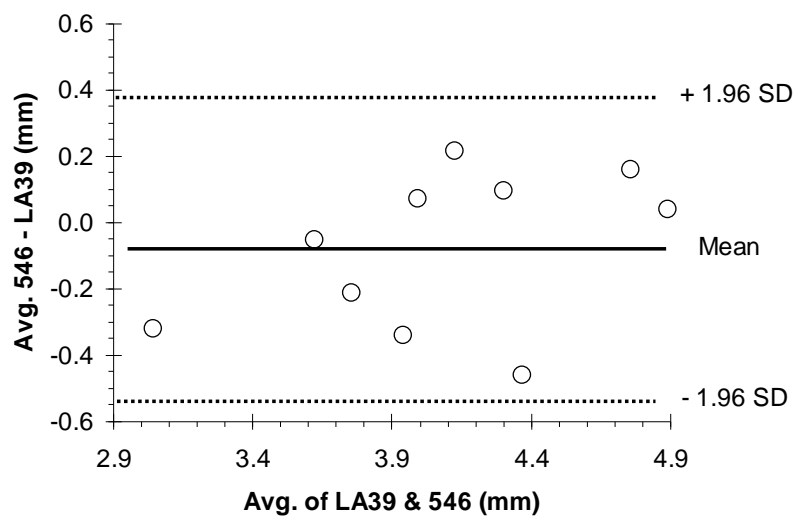

Figure 5. Bland-Altman plot of diameter difference values $(546-$ LA39) on average values $((\mathrm{LA} 39+546) / 2))$. The mean difference, upper boundary $(($ mean $+($ SD of mean $\times 1.9 .6))$, and lower boundary $(($ mean $-(\mathrm{SD}$ of mean $\times 1.96))$ are shown.

sound probe selection and optimization settings notably impact the reliability of arterial distention measurements.

\subsection{Probe Selection and Frequency Settings}

Across ultrasound settings we found that probe selection had the greatest impact on reliability for both diameter and arterial distention measurements. The lower frequency bandwidth probe $(546,6.6 \mathrm{MHz})$ resulted in bias towards smaller diameter and distention measurements. Decreasing the frequency of the LA39 probe also resulted in bias towards smaller diameter values, although this did not affect distension.

Probe selection and frequency settings may be dependent on the population sample, i.e., subjects with higher subcutaneous fat will require a probe that operates at a lower frequency bandwidth. Probe selection may also be dependent on the artery being assessed, i.e., deeper arteries will require a lower frequency bandwidth. Furthermore, during the course of an intervention study, optimal probe selection may be dependent on body compositional changes. This study shows that probe selection needs to be standardized for a given subject if repeated measures are to be made. Furthermore, diagnostic meaning for population comparisons may be influenced if different probes are used to compare populations.

\subsection{Ultrasound Imaging Mode Selection and Imaging Field}

Another important finding of this study pertains to the imaging display, i.e., B-mode vs. PD-mode (duplex). We found that PD-mode results in marginally smaller diameter and distention values. However, altering the imaging display did not affect the reliability of these measurements. We found that changing the location from a near-field to far-field did not notably impact reliability, but did result in progressive bias towards smaller diameters. The imaging display selected will be dependent on whether simultaneous blood velocity measurements are required. Reliable measurements can be made when imaging in PD-mode, however if repeated measures are to be made, then it is advisable that the imaging display remain constant. It is also advisable that image focus is maintained central to the ultrasound field.

\section{CONCLUSIONS}

Ultrasound can reliably measure arterial diameters and distension for the duration of a given test session. However, alterations to probe selection and optimization settings - particularly probe selection - can have a signifycant impact on measurement precision. Although reliability is often more important than absolute accuracy for serial exams, measurement differences due to variations in instrument settings may, nevertheless, be interpreted as having substantive diagnostic meaning. It is therefore recommended that ultrasound probe selection and optimization settings are standardized for repeated measurements, and preferably across subjects.

\section{REFERENCES}

[1] Van Popele, N.M., et al. (2001) Association between arterial stiffness and atherosclerosis: The Rotterdam study. Stroke, 32, 454-460. doi:10.1161/01.STR.32.2.454

[2] Cohn, J. (1999) Vascular wall function as a risk marker for cardiovascular disease. Journal of Hypertension, 17, S41-S44. doi:10.1016/S0895-7061(01)02154-9

[3] Cohn, J.N. (2001) Arterial compliance to stratify cardiovascular risk: More precision in therapeutic decision making. American Journal of Hypertension, 14, S258-S263. doi:10.1016/S0895-7061(01)02154-9

[4] Kingwell, B.A. (2002) Large artery stiffness: Implications for exercise capacity and cardiovascular risk. Clinical and Experimental Pharmacology and Physiology, 29, 214-217. doi:10.1046/j.1440-1681.2002.03622.x

[5] Hangiandreou, N.J., et al. (2002) The effects of irreversible JPEG compression on an automated algorithm for measuring carotid artery intima-media thickness from ultrasound images. Journal of Digital Imaging, 15, 258260.

[6] Sabatier, M.J., et al. (2006) Doppler ultrasound assessment of posterior tibial artery size in humans. Journal of Clinical Ultrasound, 34, 223-230. doi:10.1002/jcu.20229

[7] Stoner, L., et al. (2006) Upper versus lower extremity arterial function after spinal cord injury. Journal of Spinal Cord Medicine, 29, 138-146.

[8] Stoner, L., et al. (2004) The relationship between blood velocity and conduit artery diameter, and the effects of smoking on vascular responsiveness. Journal of Applied Physiology, 96, 2139-2145.

[9] Fleiss, J.L. (1986) The design and analysis of clinical experiments. Wiley, New York.

[10] Bland, J.M. and Altman, D.G. (1999) Measuring agree- 
ment in method comparison studies. Statistical Methods in Medical Research, 8, 135-160.

doi:10.1191/096228099673819272

[11] Bland, J.M. and Altman, D.G. (1986) Statistical methods for assessing agreement between two methods of clinical measurement. Lancet, 1, 307-310.

doi:10.1016/S0140-6736(86)90837-8 